\title{
Generalized Analysis Method for a Class of Novel Wideband Loaded-Stub Phase Shifters
}

\author{
Sensong AN, Badar MUNEER, Qi ZHU \\ Dept. of EEIS, University of Science and Technology of China \\ Key Laboratory of Electromagnetic Space Information, Chinese Academy of Science \\ Hefei, 230027, China \\ ass@mail.ustc.edu.cn
}

\begin{abstract}
In this paper, an analysis method of wideband loaded-stub phase shifters and a fast designing procedure is presented. These kind of phase shifters use a transmission line loaded with one or two open stubs and a reference line to achieve up to $135^{\circ}$ phase shift. Analysis results shows that lower than $-10 \mathrm{~dB}$ return loss and precisely phase shift can be achieved over a 100\% bandwidth employing the ideal open stubs. To approach the ideal impedance value of the open stubs, an arrow-shaped stub and several means to control its impedance is proposed. As verification of the analysis method, a $90^{\circ}$ two-stub loaded phase shifter is easily designed and fabricated. Measured results show the design achieves better than $\pm 4^{\circ}$ phase ripple, less than $0.5 \mathrm{~dB}$ insertion loss, and better than $10 \mathrm{~dB}$ return loss over an $85 \%$ wideband.
\end{abstract}

\section{Keywords}

Wideband, phase shifters, analysis method

\section{Introduction}

As core devices in phased array antenna system, microwave phase shifters play important roles in system performance. Broadband constant phase difference and low return loss characteristics are needed for practical applications, especially for wideband communication systems.

Several kinds of broadband phase shifters, Schiffman phase shifters [1-3], broadside coupling structure phase shifters [4], [5], transmission lines loaded with open or short stubs structures [6-8], are proposed to achieve up to $90^{\circ}$ differential phase shift. The original Schiffman phase shifters design gave a bandwidth of $80 \%$ with a phase ripple of $10^{\circ}$ [1]. In order to obtain broader bandwidth, double coupled lines and cascaded coupled lines are proposed based on the original structure [2], [3]. The main drawback of these designs is that narrow microstrip lines and extremely strict, narrow coupling gaps are required to achieve a broad bandwidth. Thus, performance of the circuit is greatly affected by fabrication accuracy. A. M. Abbosh introduced a novel UWB phase shifter structure based on weak coupling strategy, which gave a bandwidth of $110 \%$ and $\pm 3^{\circ}$ phase ripple [4], however it can only provide a phase shift range from $25^{\circ}$ to $48^{\circ}$. The structure based on the same strategy introduced in [5] increases the phase shift range to $90^{\circ}$, but phase ripple and insertion loss are also amplified $\left( \pm 10^{\circ}\right.$ and $\left.1.8 \mathrm{~dB}\right)$. Moreover this two-layer structure may cause incompatibility with other components during integration.

Loaded line phase shifters are known for its wideband characteristics, several papers were published recently. In these papers, several different designs and good results are proposed, but no clearly analysis method or designing procedures are given. In [6] a dumb-bell-shaped phase shifter is proposed, yet the dimension of the multi-section stub is determined by an optimization process. In [7] a structure composed of a transmission line loaded with shunt $\lambda / 4$ short stubs is proposed, simple relations between several differential phase degrees and resistance of the stubs are given but with no explanation. Besides, the phase ripple is quite large when the desired phase shift is more than $60^{\circ}$. In [8] a simple designing procedure for $60^{\circ}-120^{\circ}$ phase shift is given, but the resistance value is, as the paper claimed, a constant value for each degree all over the band. This designing procedure leads to not precisely phase shift, which can be proofed in the following analysis section.

In this paper, a numeric analysis method which can calculate the needful characteristics impedance of the loaded stub of all desired frequency band is presented and verified, therefore design process for all phase shifters based on loaded line structure can be largely simplified. To meet the needs of the calculation results, one arrow-shaped stub and several methods to control its impedance are given on the later part of this paper. For verification purposes, a broadband $90^{\circ}$ phase shifter employing $0.5 \lambda$ transmission line loaded with two open stubs was designed and fabricated.

\section{Analysis}

\subsection{One Stub Loaded Structure}

Generalized one stub loaded transmission line can be demonstrated in Fig. 1(a). Phase shift is obtained by calcu- 

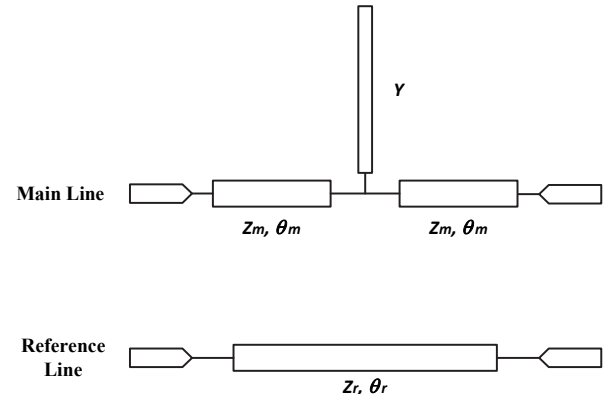

(a)

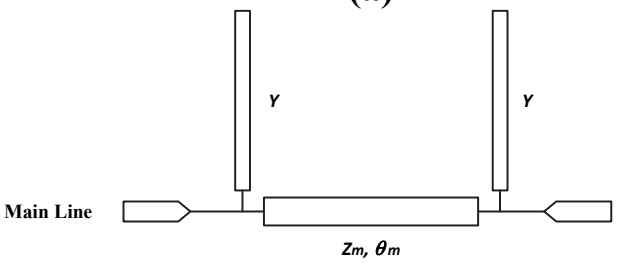

Reference

Line

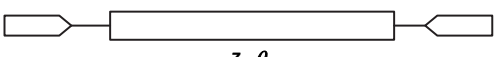

(b)

Fig. 1. Diagram of load-stub phase shifters: (a) one-stub loaded structure, (b) two-stub loaded structure.

lating the phase difference between the main line and the reference line. Assuming that characteristic impedance and electric length is $Z_{\mathrm{m}}, \theta_{\mathrm{m}}$ for the main line and $Z_{\mathrm{r}}, \theta_{\mathrm{r}}$ for the reference line, admittance of the loaded stub is $j Y$, electrical length of the transmission line and reference line at frequency $f$ are defined by center frequency $f_{0}$ :

$$
\theta_{\mathrm{m}}=\theta_{\mathrm{m} 0} \frac{f}{f_{0}}, \quad \theta_{\mathrm{r}}=\theta_{\mathrm{r} 0} \frac{f}{f_{0}} .
$$

Then the transfer matrix of the main line can be defined as [9]:

$\left[\begin{array}{cc}\cos \theta_{\mathrm{m}} & j Z_{\mathrm{m}} \sin \theta_{\mathrm{m}} \\ j \frac{1}{Z_{\mathrm{m}}} \sin \theta_{\mathrm{m}} & \cos \theta_{\mathrm{m}}\end{array}\right]\left[\begin{array}{cc}1 & 0 \\ j Y & 1\end{array}\right]\left[\begin{array}{cc}\cos \theta_{\mathrm{m}} & j Z_{\mathrm{m}} \sin \theta_{\mathrm{m}} \\ j \frac{1}{Z_{\mathrm{m}}} \sin \theta_{\mathrm{m}} & \cos \theta_{\mathrm{m}}\end{array}\right]$.

Thus

$$
\begin{gathered}
A=D=\cos \left(2 \theta_{\mathrm{m}}\right)-0.5 Z_{\mathrm{m}} Y \sin \left(2 \theta_{\mathrm{m}}\right), \\
B=j Z_{\mathrm{m}} \sin \left(2 \theta_{\mathrm{m}}\right)-j Z_{\mathrm{m}}^{2} Y \sin ^{2}\left(\theta_{\mathrm{m}}\right) \\
C=j \frac{1}{Z_{\mathrm{m}}} \sin \left(2 \theta_{\mathrm{m}}\right)+j Y \cos ^{2}\left(\theta_{\mathrm{m}}\right) .
\end{gathered}
$$

According to transformational relations between transfer matrix and S-parameters, S11, S21 and phase shift between port 1 and port 2 can be derived through $A B C D$ value [9]:

$$
S_{11}=S_{22}=\frac{A+\frac{B}{Z_{\mathrm{m}}}-C Z_{\mathrm{m}}-D}{A+\frac{B}{Z_{\mathrm{m}}}+C Z_{\mathrm{m}}+D}=\frac{\frac{B}{Z_{\mathrm{m}}}-C Z_{\mathrm{m}}}{2 A+\frac{B}{Z_{\mathrm{m}}}+C Z_{\mathrm{m}}}
$$

$$
\begin{gathered}
S_{12}=S_{21}=\frac{2}{A+\frac{B}{Z_{\mathrm{m}}}+C Z_{\mathrm{m}}+D}=\frac{2}{2 A+\frac{B}{Z_{\mathrm{m}}}+C Z_{\mathrm{m}}}, \\
\Delta \Phi=\theta_{\mathrm{r}}-\tan ^{-1}\left(\frac{\frac{B}{Z_{\mathrm{m}}}+C Z_{\mathrm{m}}}{2 A}\right) .
\end{gathered}
$$

In order to derive the ideal performance at center frequency, which means, $S_{11}=0$ and $S_{21}=1$, then $\theta_{\mathrm{m} 0}=\pi / 2$ can be determined from (2), (3) and (4). The value of $Z_{\mathrm{m}}$ is taken as $50 \Omega$, therefore, the electrical length of the reference line $\theta_{\mathrm{r} 0}$ is simply given by,

$$
\theta_{\mathrm{r} 0}=\Delta \Phi+\theta_{\mathrm{m} 0}=\Delta \Phi+\frac{\pi}{2}
$$

where $\Delta \Phi$ is the phase difference between the two lines and is given by

$$
\Delta \Phi=\theta_{r}-\tan ^{-1}\left(\frac{2 \sin \left(2 \theta_{\mathrm{m}}\right)+Z_{\mathrm{m}} Y \cos \left(2 \theta_{\mathrm{m}}\right)}{2 \cos \left(2 \theta_{\mathrm{m}}\right)-Z_{\mathrm{m}} Y \sin \left(2 \theta_{\mathrm{m}}\right)}\right) .
$$

In the above expression, all the parameters are known except the admittance $Y$. For the frequency band from $0.5 f_{0}$ to $1.5 f_{0}$, to obtain flat phase response (e.g. $22.5^{\circ}, 45^{\circ}$ and $90^{\circ}$ ), the ideal admittance $Y$ of the stubs can be calculated by using (7), as shown in Fig. 2(a).

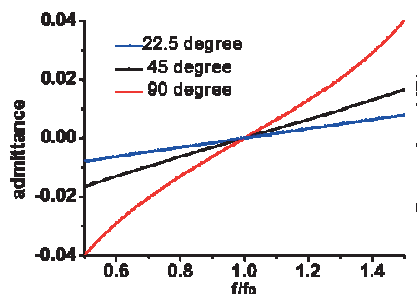

(a)

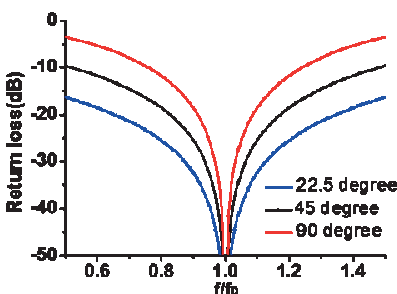

(b)
Fig. 2. One stub calculated results: (a) ideal admittance, (b) return loss calculated with ideal admittance.

Once the admittance $Y$ is obtained, it is straight-forward to get the expression for return loss of the main line given by (3):

$$
\begin{aligned}
S_{11}= & \frac{-j Y Z_{\mathrm{m}}}{2 \cos \left(2 \theta_{\mathrm{m}}\right)-Y Z_{\mathrm{m}} \sin \left(2 \theta_{\mathrm{m}}\right)+} \\
& j\left[2 \sin \left(2 \theta_{\mathrm{m}}\right)+Y Z_{\mathrm{m}} \cos \left(2 \theta_{\mathrm{m}}\right)\right]
\end{aligned}
$$

The plot for $S_{11}$ obtained using (8) is shown in Fig. 2(b). The plot shows wideband characteristics for smaller values of phase shift, and deteriorates to $40 \%$ bandwidth when the phase shift reaches up to 90 degrees.

\subsection{Two Stub Loaded Structure}

Generalized two stub loaded transmission line can be demonstrated as Fig. 1(b). Following the same procedure for single stub loaded transmission line, its transfer matrix can be defined as: 


$$
\begin{gathered}
{\left[\begin{array}{cc}
1 & 0 \\
j & 1
\end{array}\right]\left[\begin{array}{cc}
\cos \theta_{\mathrm{m}} & j Z_{\mathrm{m}} \sin \theta_{\mathrm{m}} \\
j \frac{1}{Z_{\mathrm{m}}} \sin \theta_{\mathrm{m}} & \cos \theta_{\mathrm{m}}
\end{array}\right]\left[\begin{array}{cc}
1 & 0 \\
j Y & 1
\end{array}\right],} \\
A=D=\cos \theta_{\mathrm{m}}-Z_{\mathrm{m}} Y \sin \theta_{\mathrm{m}} \\
B=j Z_{\mathrm{m}} \sin \theta_{\mathrm{m}}, \\
C=2 j Y \cos \theta_{\mathrm{m}}+j \frac{1}{Z_{\mathrm{m}}} \sin \theta_{\mathrm{m}}-j Y^{2} Z_{\mathrm{m}} \sin \theta_{\mathrm{m}}
\end{gathered}
$$

Putting the $S_{11}=0$ and $S_{21}=0$ results in the value for $\theta_{\mathrm{m} 0}=\pi$, the electrical length of the reference line is therefore given by

$$
\theta_{\mathrm{r} 0}=\Delta \Phi+\theta_{\mathrm{m} 0}=\Delta \Phi+\pi .
$$

Similarly, the expression for $\Delta \Phi$ can be obtained in terms of unknown admittance $Y$ for $Z_{\mathrm{m}}=50 \Omega$

$$
\begin{array}{r}
\Delta \Phi=\theta_{r}-\tan ^{-1}\left(\begin{array}{c}
2 \sin \theta_{\mathrm{m}}+2 Z_{\mathrm{m}} Y \cos \theta_{\mathrm{m}} \\
\frac{-Y^{2} Z_{\mathrm{m}}^{2} \sin \theta_{\mathrm{m}}}{2 \cos \theta_{\mathrm{m}}-2 Z_{\mathrm{m}} Y \sin \theta_{\mathrm{m}}}
\end{array}\right), \\
S_{11}=\frac{j Y^{2} Z_{\mathrm{m}}^{2} \sin \theta_{\mathrm{m}}-2 j Y Z_{\mathrm{m}} \cos \theta_{\mathrm{m}}}{2 \cos \theta_{\mathrm{m}}+2 j \sin \theta_{\mathrm{m}}-2 Y Z_{\mathrm{m}} \sin \theta_{\mathrm{m}}} . \\
\quad+j Y Z_{\mathrm{m}}\left(2 \cos \theta_{\mathrm{m}}-Y Z_{\mathrm{m}} \sin \theta_{\mathrm{m}}\right)
\end{array}
$$

For the band from $0.5 f_{0}$ to $1.5 f_{0}$, the ideal admittance $Y$ is calculated using (11), the return loss is calculated using (12), as given in Fig. 3(a) and Fig. 3(b), respectively.

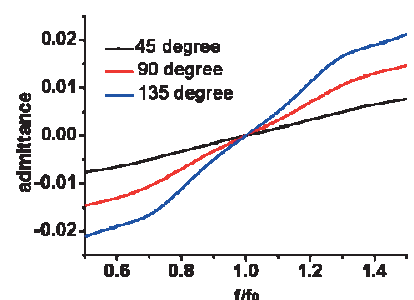

(a)

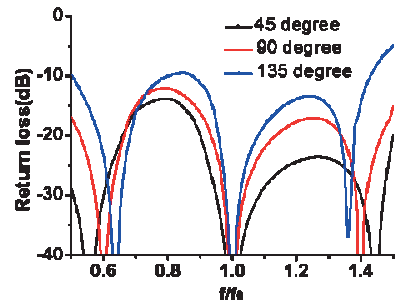

(b)
Fig. 3. Two stub calculated results: (a) ideal admittance, (b) return loss calculated with ideal admittance.

Figure 3(b) also shows good wideband characteristics. Compared to single stub loaded structure, two stub loaded structure shows better return loss when larger differential phase shift is obtained.

\section{Comparison and Validation}

To verify the reliability of the proposed analysis method, two $90^{\circ}$ phase shifters loaded with simple rectangular stubs, which have same electrical length $\pi$ and different characteristic impedances are designed and simulated. Both phase shifters have center frequency of $4 \mathrm{GHz}$. Blue curve in Fig. 4(a) refers to the calculated admittance, black and red curves refer to admittance of stubs using $150 \Omega$ and $200 \Omega$ transmission lines, respectively. Simulation results

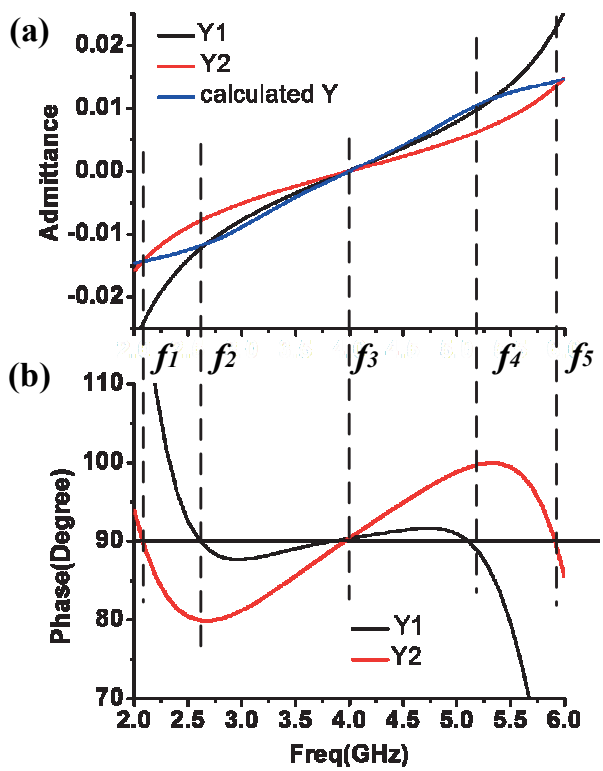

Fig. 4. Relations between admittance of the stubs and phase shift.

are shown in Fig. 4(b). At discrete frequency values $f_{1}, f_{3}$ and $f_{5}$ the admittances of the $200 \Omega$ line can be seen to be equal to the calculated ones, resulting in a precise phase shift of $90^{\circ}$. At discrete frequency values $f_{2}, f_{3}$ and $f_{4}$ the admittances of the $150 \Omega$ line become equal to the calculated ones, resulting in $90^{\circ}$ phase shift. For the whole $100 \%$ band, positive correlation between phase ripple and admittance deviation can be seen, which strongly validates the reliability and accuracy of the proposed analysis method.

\section{Application}

\subsection{Arrow-Shaped Stub}

To fit better to the calculated admittance, a method to enlarge the impedance of the stubs is presented.

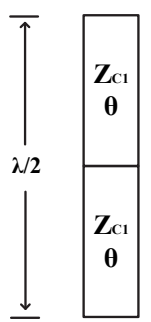

(a)

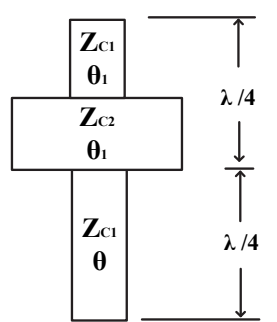

(b)
Fig. 5. Diagram of two stubs: (a) rectangular stub. (b) stepped rectangular stub.

According to transmission line impedance equation:

$$
Z=Z_{0} \frac{Z_{\mathrm{L}}+j Z_{0} \tan \theta}{Z_{0}+j Z_{\mathrm{L}} \tan \theta}=\frac{Z_{\mathrm{L}}+j Z_{0} \tan \theta}{1+j \frac{Z_{\mathrm{L}}}{Z_{0}} \tan \theta}
$$

the admittance of a signal stub is obtained: 


$$
Y=\frac{1+j \frac{Z_{\mathrm{L}}}{Z_{0}} \tan \theta}{Z_{\mathrm{L}}+j Z_{0} \tan \theta}=j\left(\frac{\tan \theta}{Z_{0}}-\frac{1+\tan ^{2} \theta}{Z_{\mathrm{L}}+Z_{0} \tan \theta}\right) .
$$

To lower the admittance, smaller loaded impedance $Z_{\mathrm{L}}$ is needed.

By changing the shape of loaded lines from rectangular patches (Fig. 5(a)) into stepped rectangular patches (Fig. 5(b)), its impedance can be derived as:

$$
Z_{\mathrm{L}}=Z_{\mathrm{C} 2} \frac{Z_{1}+j Z_{\mathrm{C} 2} \tan \theta_{1}}{Z_{\mathrm{C} 2}+j Z_{1} \tan \theta_{1}}
$$

where

$$
Z_{1}=\frac{Z_{\mathrm{C} 1}}{j \tan \theta_{1}}
$$

So

$$
Z_{\mathrm{L}}=j \frac{Z_{\mathrm{C} 2} \tan \theta_{1}-\frac{Z_{\mathrm{C} 1}}{\tan \theta_{1}}}{1+\frac{Z_{\mathrm{C} 1}}{Z_{\mathrm{C} 2}}}=\frac{Z_{\mathrm{C} 1}\left(1-\frac{Z_{\mathrm{C} 2}}{Z_{\mathrm{C} 1}} \tan ^{2} \theta_{1}\right)}{j \tan \theta_{1}\left(1+\frac{Z_{\mathrm{C} 1}}{Z_{\mathrm{C} 2}}\right)} .
$$

Compared to the impedance of rectangular patches:

$$
Z_{\mathrm{L}}^{\prime}=\frac{Z_{\mathrm{C} 1}}{j \tan \theta}=\frac{Z_{\mathrm{Cl}}}{j \tan \left(2 \theta_{1}\right)}=\frac{Z_{\mathrm{Cl}}\left(1-\tan ^{2} \theta_{1}\right)}{2 j \tan \theta_{1}} .
$$

Comparing (17) with (18), cause $Z_{\mathrm{C} 1}>Z_{\mathrm{C} 2}, Z_{\mathrm{L}}<Z_{\mathrm{L}}$ can be concluded. Thus it's possible to use a transmission line loaded with stepped rectangular patch to replace the regular rectangular $\lambda / 4$ stub to lower the admittance. By the mean time very high impedance transmission line is no longer needed, which also lowers the difficulty of the fabrication.

According to the analysis above, an arrow-shaped stub is presented, as shown in Fig. 6(a). Compared to simple rectangular stubs, low admittance (high resistance) is

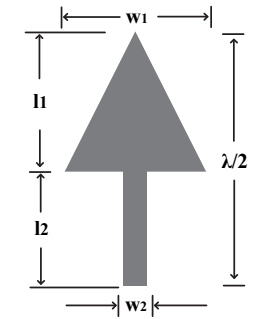

(a)

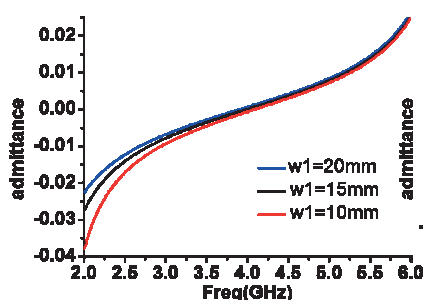

(c)

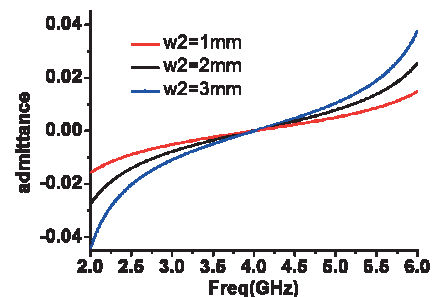

(b)
Fig. 6. Arrow-shaped stub and methods to control its admittance: (a) diagram of arrow-shaped stub, (b), (c) and (d) methods to control its admittance over the band. much easier to achieve. Besides, admittance value of the proposed stub is able to be easily controlled by several means, as shown in Fig. 6(b), (c) and (d). The whole length of the stub is fixed to be $\lambda / 2$, to ensure low return loss at center frequency.

\subsection{Design Example and Results}

As an example, a $90^{\circ}$ degree phase shifter loaded with two arrow-shaped stubs is designed and fabricated. Admittance of the stubs is designed to be highly close to the ideal values all over the band, which offers a precise constant phase shift. Measured results shown in Fig. 7 achieve better than $13 \mathrm{~dB}$ return loss, $0.5 \mathrm{~dB}$ insertion loss and phase error less than $4^{\circ}$ over a wide bandwidth $(2.5 \mathrm{GHz}$ to $6 \mathrm{GHz}$ ). The overlapping results shown in Fig. 7 also indicate good matching between measured results and simulated results both in S-parameters and phase shift.

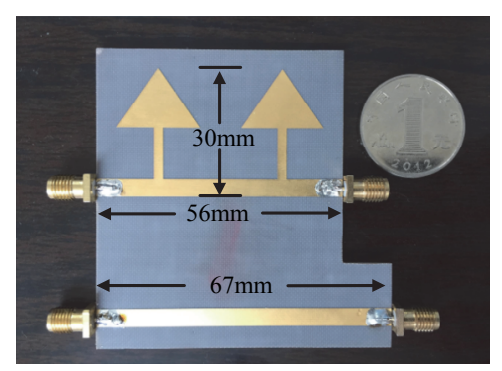

(a)

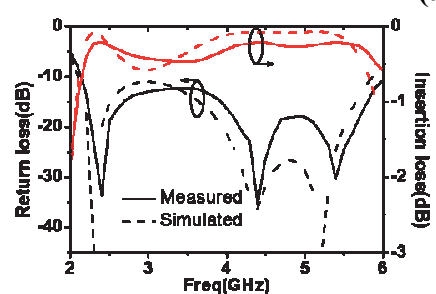

(b)

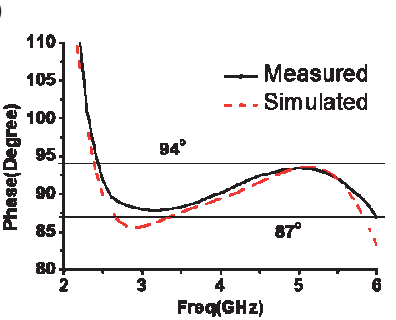

(c)
Fig. 7. Simulated and measured results: (a) one of the manufactured phase shifters, (b) S-parameters, (c) phase shift.

\subsection{Loss Mechanism}

The losses associated with dielectric material as well as the conductor loss have a certain effect on the insertion loss of phase shifter, a perfect conductor and a dielectric material with no loss always lead to higher return loss, this is clear from Fig. 7(b). To further elaborate the interferences of these losses, several simulations are performed with different scenarios.

Figure 8(a) and (b) show the effect of dielectric losses. With larger dielectric loss tangent $(\tan \delta)$, insertion losses increase while return losses remain almost the same. Figure 8(c) shows the effect of conductor losses; with larger resistivity, matching properties deteriorate slightly while insertion losses increase rapidly, about $0.1 \mathrm{~dB}$ across the band when the conductor changes from PEC to copper.

Taking into account all the losses, let the dielectric 
loss tangent be 0.001 and conductivity be $5.6 \mathrm{e} 8$ Siemens (provided by the PCB manufacture), renewed simulation results are shown in Fig. 8(d). Compared to ideal simulation results shown in Fig. 7(b), both curves fit better with the measured results.

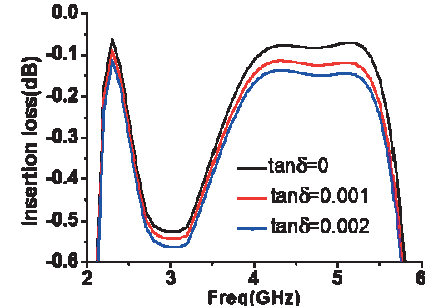

(a)

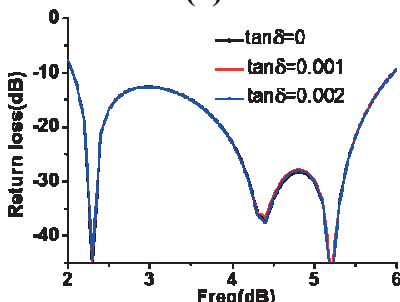

(b)

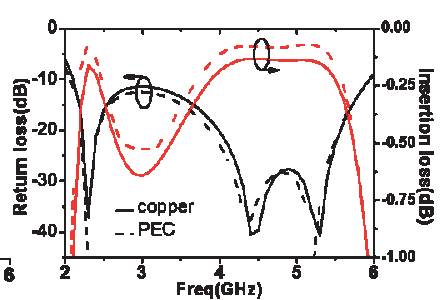

(c)

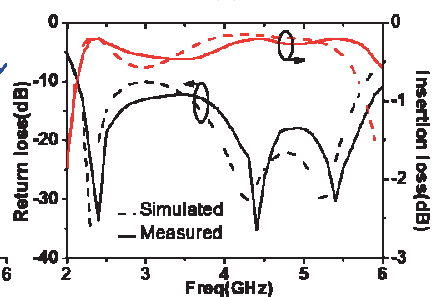

(d)

Fig. 8. Loss discussion: (a) insertion loss with different dielectric loss tangent, (b) return loss with different dielectric loss tangent, (c) S-parameters with different conductor losses, (d) renewed simulated results.

\section{Conclusion}

This paper reports on an analysis method of a class of novel wideband phase shifters. According to this method, phase shift up to 45 degrees using a single stub-loaded structure and phase shift up to 135 degrees employing a two stub-loaded structure can be achieved over an $85 \%$ of bandwidth with phase error below $\pm 4^{\circ}$. Moreover, a fast design procedure is presented, therefore the design process for all phase shifters based on loaded line structure is largely simplified. For verification purpose, a $90^{\circ}$ phase shifter is designed, fabricated and measured.

\section{Acknowledgments}

This work is supported by the National Natural Science Foundation of China under Grant No.61131002.

\section{References}

[1] SCHIFFMAN, B. M. A new class of broad-band microwave 90degree phase shifters. IEEE Transactions on Microwave Theory and Techniques, Apr. 1958, vol. 6, no. 2, p. 232-237. DOI: 10.1109/TMTT.1958.1124543

[2] QUIRARTE, J. L. R., STARSKI, J. P. Novel Schiffman phase shifters. IEEE Transactions on Microwave Theory and Techniques, 1993, vol. 41, no. 1, p. 9-14. DOI: 10.1109/22.210223

[3] GUO, Y. X., ZHANG, Z. Y., ONG, L. C. Improved wide-band Schiffman phase shifter. IEEE Transactions on Microwave Theory and Techniques, 2006, vol. 54, no. 3, p. 1196-1199. DOI: 10.1109/TMTT.2005.864105

[4] ABBOSH, A. M. Ultra-wideband phase shifters. IEEE Transactions on Microwave Theory and Techniques, Sep. 2007, vol. 55, no. 9, p. 1935-1941. DOI: 10.1109/TMTT.2007.904051

[5] WANG, Y., BIALKOWSKI, M. E., ABBOSH, A. M. Double microstrip-slot transitions for broadband $\pm 90^{\circ}$ microstrip phase shifters. IEEE Microwave Wireless. Components Letters, Feb. 2012, vol. 22, no. 2, p. 58-60. DOI: 10.1109/LMWC.2011.2181348

[6] ZHENG, S. Y., YEUNG, S. H., CHAN, W. S., MAN, K. F., LEUNG, S. H. Improved broadband dumb-bell-shaped phase shifter using multi-section stubs. Electronics Letters, 2008, vol. 44, no. 7 , p. 478 . DOI: $10.1049 / \mathrm{el}: 20083364$

[7] TANG, X., MOUTHAAN, K. Design of a UWB phase shifter using shunt lambda/4 stubs. In IEEE MTT-S International Microwave Symposium Digest, 2009, p. 1021-1024. DOI: 10.1109/MWSYM.2009.5165873

[8] ZHENG, S. Y., CHAN, W. S., MAN, K. F. Broadband phase shifter using loaded transmission line. IEEE Microwave Wireless Components Letters, 2010, vol. 20, no. 9, p. 498-500. DOI: 10.1109/LMWC.2010.2050868

[9] POZAR, D. M. Microwave Engineering. $3^{\text {rd }}$ ed. John Wiley \& Sons, 2005, p. 728 . ISBN: 8126510498

\section{About the Authors ...}

Sensong AN was born in Tianjin, China. He received his undergraduate degree from USTC in 2013. His research interests include microwave components and antennas.

Badar MUNEER was born in Mirpurkhas, Pakistan in 1987. He received his B.S degree in Communication Systems Engineering from the Institute of Space Technology, Islamabad, Pakistan, in 2008 and the M.Engg Degree in Telecommunication Engineering from NED University of Engineering and Technology, Karachi, Pakistan, in 2012. $\mathrm{He}$ is currently pursuing the Ph.D. degree in Electromagnetic Field and Microwave Technology at the University of Science and Technology of China (USTC), Hefei, P.R China. From 2008 to 2011, he was with a satellite broadcasting company as a satellite engineer. He worked on VSAT, CATV and many modern broadcast equipment. His current research interests are in the area of microwave and millimeter-wave technology, SIW based power dividers and phase shifters.

Qi ZHU received the B.S degree and M.S degree in Dept. of Physics from Hefei Univ. of Technology, China, in 1989 and 1992, and received Ph.D. in Dept. of Airplane from Nanjing University of Aeronautics and Astronautics. He is now working as a professor in the University of Science and Technology of China. His research interests are in the area of microwave and millimeter-wave technology. 